\title{
An Efficient Gravitational Search Algorithm Based Optimal Web Service Selection for Composition in
}

\section{SOA}

\author{
D.Palanikkumar \\ Department of CSE \\ Anna University of Technology \\ Coimbatore
}

\author{
P.Anbuselvan \\ Department of CSE \\ Anna University of Technology \\ Coimbatore
}

\author{
M.Kathiravan \\ Department of CSE \\ Anna University of Technology \\ Coimbatore
}

\begin{abstract}
The Web services model has emerged as a standard for representation, discovery, and invocation of services in a distributed environment. During service composition, Selection of appropriate services for composition is the chief task. A series of tasks are tied together to form a composite web service. Such composition is a challenging task because a number of Web services with the same or similar functions are increasing rapidly. QoS plays a vital role in the problem of selecting the most appropriate web service for composition. It is a measure for how well the composite web service serves the requester. Particle Swarm Optimization (PSO) algorithm and Gravitational Search Algorithm can be used to resolve this problem of optimal service selection. To compose individual web services dynamically according to users' requirements in functional aspects as well as in non-functional preferences. In order to meet the QoS requirements of consumers, this paper presents the QoS calculation of non-functional requirements such as cost, availability, reliability and execution time. To verify the effectiveness in latency of Web Services selection the above two algorithms are compared. Results indicate the Gravitational Search Algorithm improves the latency over the Particle Swarm Optimization algorithm.
\end{abstract}

\section{Keywords: PSO, GSA, Service Selection.}

\section{INTRODUCTION}

The Web services model has emerged as a standard for representation, discovery, and invocation of services in a distributed environment. During service composition, Selection of appropriate services for composition is the chief task. Many sophisticated tools are required for a web consumer to search for the best service that satisfies his needs. Several web services may exist that provide the same functionality. In such a case, Quality of Service (QoS) is the decisive factor in distinguishing the functionally similar services. QoS-aware Web Service composition is defined as the selection of Web Services maximizing the QoS of the overall Web Service composition, taking into account preferences and constraints defined by the user. Web service composition is gaining a considerable momentum as an approach to the effective integration of distributed, heterogeneous, and autonomous application[12].The process is to search for the optimal set of services that can be composed to create a new service, result in the best QoS with user constraints [2, 18]. Web service has three basic underlying components which form its basic platform; WSDL, UDDI and SOAP [17, 12]. These components are used to select the suitable web services based on client's request. WSDL (Web Service Description Language) is an XML based language. It is used to describe the web services with its functionalities and provides an end point to invoke the web service. A web service is described in the standard XML format of service description using WSDL. It has similar purpose of IDLs (Interface Definition Language) [11, 5].
UDDI have enabled service providers and requesters to find the web service through UDDI Business Registries [20, 4]. In order to select a suitable web services that are published and made available to the clients, every web service must initially publish in some UDDI registries [1, 5].SOAP defines how to communicate the information using XML in web service selection. SOAP specifies the message format and description of message should be transported using HTTP and SMTP [3, 5]. Recently Service selection algorithms are mainly genetic algorithm, Ant colony algorithm, linear programming, and Particle Swarm algorithm and so on [16]. An efficient method is PSO-based service Selection which can be performed by considering user's functional and QoS constraints. Several algorithms exist to perform this chief task of Service selection based on QoS. Each service that exists for accomplishing a specific task can be considered as a particle. So several particles may exist, thus forming a population.PSO can then be applied to find the best solution among the candidates to form an optimal composition. An optimization algorithm called Particle Swarm Optimization (PSO) can be applied to solve this problem of Service selection for composition. Gravitational Search Algorithm is used in an NP hard problem area in a large power system. This optimization deals to find the best location of SVC (Static Var Compensator). The optimization is made on two parameters: location and size [6]. The problem of web service selection for composition can be defined as follows, "In an algorithm based approach of web service selection, which evolutionary search and optimization technique can obtain the best result". 
This paper presents a comparative approach to Service selection for Service Composition based on QoS with the user's constraints. Gravitational Search Algorithm (GSA) is one of the optimization algorithms based on law of gravity $[19,3]$.

\section{WEB SERVICE COMPOSITION}

Web Service composition aims at selecting and interconnecting Web Services provided by different partners according to a business process. Thus, Web Service compositions can be seen as workflows based on Web Services. There is a workflow model that consists of abstract tasks describing the required functionality (e.g. invoking a credit rating) of a specific workflow step. One of the main issues hereby is the selection of appropriate Web Services that form the execution plan for a Web Service composition. The functionality of each task can be provided by different candidate Web Services. Web Services that provide similar or identical functionality are grouped in the same category [7]. Web Services within the same category may have different non-functional attributes. Fig.1 explains Web Services has many service oriented application. It is one of the web service compositions and selects the best service in the value added services. Existing technologies of web services are extended to give value added customized services to customers through composition. If no single Web service can satisfy the functionality required by the user, there should be a possibility to combine existing services together in order to fulfill the request. The basic web service model consists of three entities: Service Provider, Service Registry and Service Requester [12]. Service Provider is used to provide the service to the Service Registry [5]. Service Requester is the requester who retrieves the information from service registry to find a suitable service provider and publish the web service. The Service Registry contains the information about Service Provider and Service Requester [10].

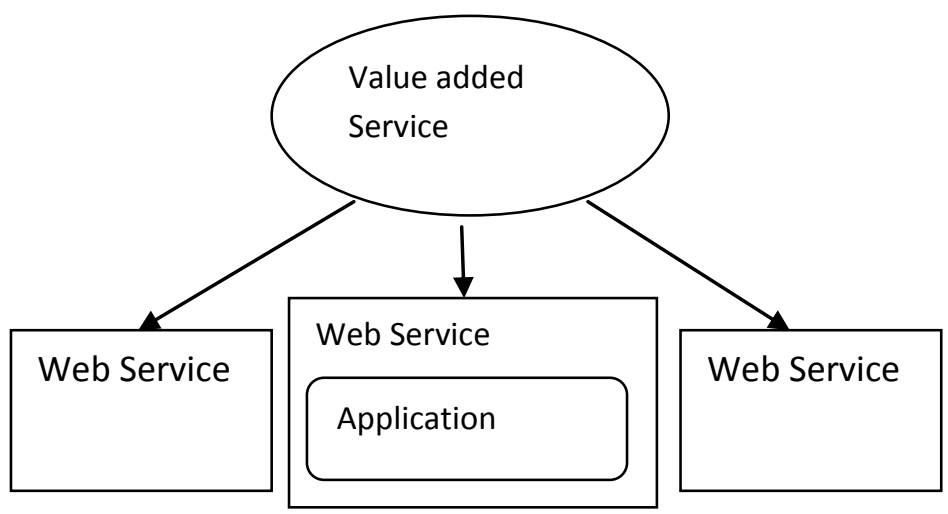

Fig.1 Selection of Web Service Application

Execution cost: The execution cost of an operation of a service is the fee that a service requester has to pay for invoking the service and executing its operation.

Response Time: The response time measures the maximum delay in seconds between the moment when a request is sent and the moment when the results are received by client-side view.
Reliability: The reliability is a measure of trustworthiness of a service. It measures the degree of compliance between providers claimed value with the actual value.

Availability: The availability is the probability that the service is accessible. It is the quality aspect of whether the service is available for immediate use.

\subsection{The web services selection problem}

Motivating Example: In this section, we present a travel plan domain such as hotel, airline and taxi reservation web service that receives information regarding the type of customer wishes to reserve and makes a reservation to customer needs. Each web service have four individual Services such as Service1, Service2, Service 3, and Service 4.Using the both algorithm we can select the best Services in the Input Details. Fig. 2 has shown the diagram for this model. This study describes how Particle Swarm Optimization Algorithm and Gravitational Search Algorithm can be applied to the optimization problem of optimal web service selection and compares the performance of both the algorithms.

\section{WEB SERVICE SELECTION BASED ON PSO ALGORITHM}

PSO Algorithm introduced by Kennedy and Eberhart in 1995[1]. The Particle Swarm Optimization Algorithm based on user Preferences in web service selection [7]. In this Paper, we mainly solved service selection problem in the web service composition. The PSO-based web service selection method (PSOWSS) to resolve dynamic web services selection with global QoS constraints, considering various QoS attributes, such as response time, cost, and availability etc.

\section{Algorithm}

Input: target function;

Output: Pareto solutions

1) Initialization set up parameters;

2) In accordance with population size set, randomly generated paths of service composition to meet the constraint. Each path is encoded as a particle, and all particles form the initial particle population;

3) Implementing the disturbance moving of particles;

4) Randomly selecting current number of mutation particles( PN mutation) from external population, and updating those;

5) Updating the values of pbest and gbest;

6) According to the evolution times of fitness function to judge whether meeting the end conditions or not. 


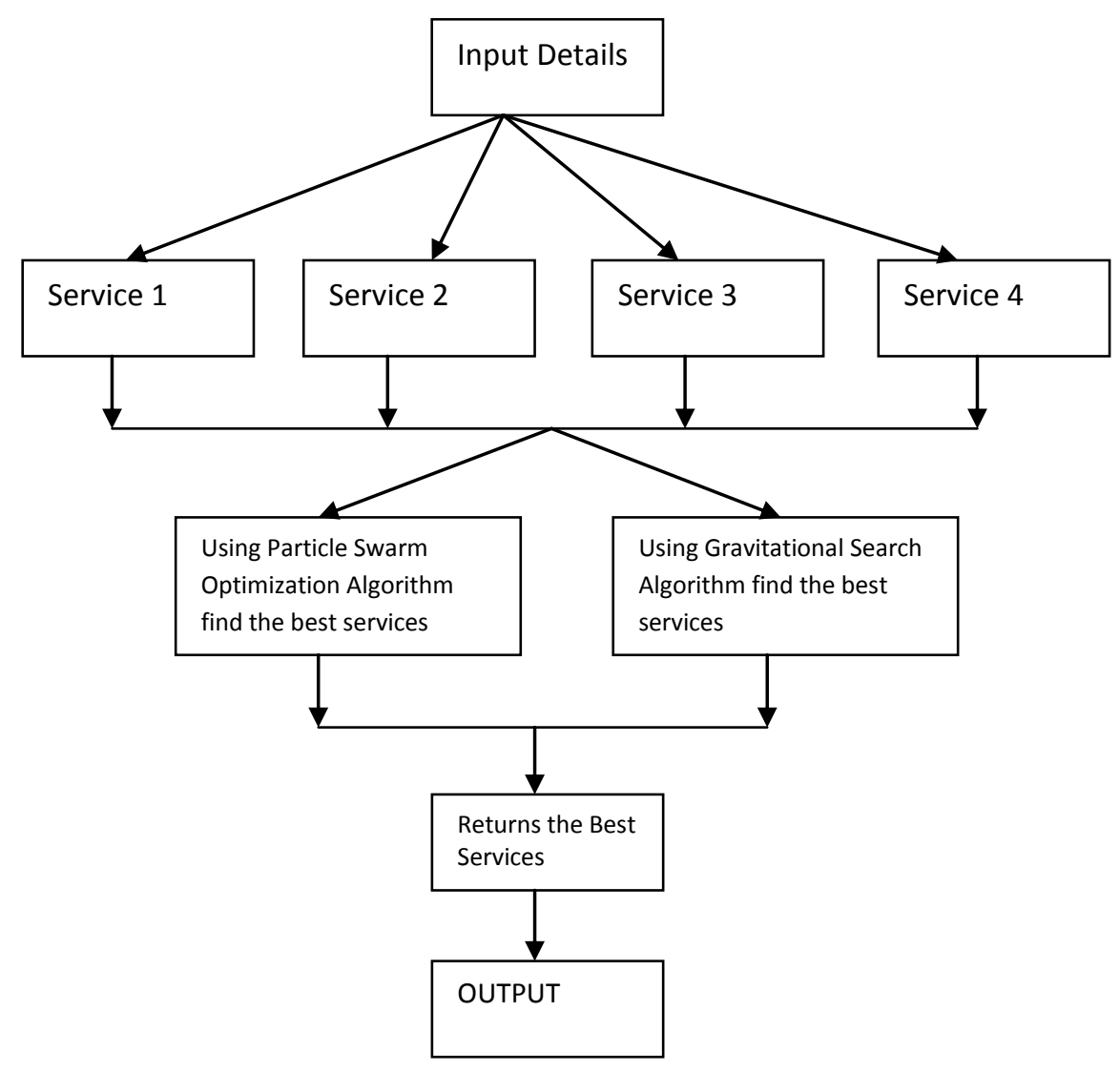

Fig.2 Travel plan domain

Generating Initial Particle Population

Input: population size $(\mathrm{N})$;

Output: initial particle population (SN)

1) Set SP to empty;

2) Add the sets that are generated by Constr(RanService(WS)) into SP $|\mathrm{SP}|=|\mathrm{SP}|+\mathrm{I}$;

3) If $|\mathrm{SP}|=\mathrm{N}$ then goto 2 else ending;

4) Where WS is the set of service candidates; RanService(WS) is randomly service path generating method and its range is service selection scenarios; Constr( ) is service selection method based on constraints.

\section{WEB SERVICE SELECTION BASED ON GRAVITATIONAL SEARCH ALGORITHM}

Gravitational Search Algorithm is based on the law of gravity. It Consider agents as objects and their performance measured by their masses.

Now, consider the travel plan domain created, which is a web service application for flight, hotel and taxi Reservation. The application uses non functional requirements such as price, duration, reputation, rate and availability. Each service has four candidate services.

The fitness value of each service is calculated in web application using the non functional parameter and stored into the new table.
Gravitational and inertial are calculated by the fitness evaluation. The gravitational and inertial masses are updating by the following equations:

$\mathrm{Mg}_{\mathrm{i}}=(\operatorname{fit}(t)-\operatorname{Worst}(t)) /(\operatorname{Best}(t)-\operatorname{Worst}(t))$

Here fit $(\mathrm{t})$ calculated by the non functional requirement parameters and represents the fitness value of the each services at time $t$.

Fit $t$

$=\frac{(\text { Qprice }+ \text { Qduration }+ \text { Qreputation }+ \text { Qrate }+ \text { Qavailability })}{\text { Qtime }}$

Where fit(t) represent the fitness value of the agents $i$ at time $t$, worst $(t)$ and best $(t)$ are defined as follows:

Best $(\mathrm{t})=$ Max fit $(\mathrm{t})$

Worst $(\mathrm{t})=$ Min fit $(\mathrm{t})$

At a specific time' $t$ ', the fitness value is calculated by:

$$
\text { Fit } t=\frac{(g t * M g i)}{(\operatorname{Rij} t+e s)} * \text { Fit }(t)
$$

Here gt is gravitational constant at time $\mathrm{t}$, es is small constant. fitness is fit $(\mathrm{t})$ value of each services.

$\mathrm{R}_{\mathrm{ij}}(\mathrm{t})=(\text { euclidian } 1+\text { euclidian } 2)^{2}$

$\mathrm{R}_{\mathrm{ij}}(\mathrm{t})$ is the Euclidian distance between two services. Here we can calculate the Euclidian1 using the flight and hotel services and Euclidian2 using the Euclidian1 and car services. Euclidian1 $=(\text { flight service }- \text { hotel service })^{2}$ Euclidian 2= (flight service - taxi service $)^{2}$ 


\begin{tabular}{l|llll|ll}
\hline Execution & Parameters & & & & \multicolumn{2}{l}{ Latency(ms) } \\
\hline$\#$ & Availability & Reliability & Cost & Reputation & PSO & GSA \\
\hline 1 & $\{0,1\}$ & $\{0,1\}$ & Varied & Ranked & 375 & 156 \\
2 & $\{0,1\}$ & $\{0,1\}$ & Varied & Ranked & 140 & 109 \\
3 & $\{0,1\}$ & $\{0,1\}$ & Varied & Ranked & 109 & 125 \\
4 & $\{0,1\}$ & $\{0,1\}$ & Varied & Ranked & 125 & 93 \\
5 & $\{0,1\}$ & $\{0,1\}$ & Varied & Ranked & 375 & 109 \\
\hline
\end{tabular}

Table 1 Comparison between PSO and GSA

\section{EXPERIMENTAL RESULTS}

\subsection{Comparing the Fitness Functions}

In this paper we compared the fitness functions of Particle Swarm Optimization algorithm and Gravitational Search algorithm. In PSO algorithm updating is performed without considering the fitness value. In GSA, fitness value is important around the search space. In GSA fitness is reversely proportional to the distance between solutions. Particle Swarm Optimization algorithm Calculates the fitness values using Group of iteration,then compute the best values from the global values of iteration. So the time consumption is more. But, in GSA, choose fitness as random iteration of web service for calculate the fitness value. So the time consumption much lower compared to Particle Swarm Optimization algorithm

\subsection{Comparing PSO with GSA}

The comparison results shown in Fig.3.For the particular time $t$, Gravitational Search algorithm performs better than PSO algorithm. Gravitational Search algorithm solving the NP-hard problem like web service selection better than PSO algorithm. This proves all the particle be likely to converge to the best solution quickly compared to Particle Swarm Optimization algorithm. This is represented in the figure.(Refer Table 1)

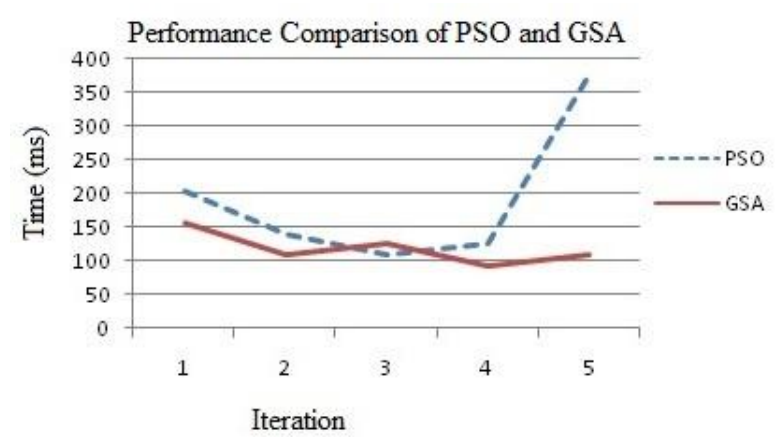

Fig. 3 Comparison of PSO vs GSA

\section{CONCLUSION}

This Paper explains the web service selection problem by considering a travel plan domain as an example. The algorithm can generate a set of optimal plan to meet user constraints. We have presented an algorithmic approach for solving the optimal service selection optimization problem by considering two optimization algorithm, PSO and GSA algorithm. PSO-based web service selection selects the optimal Web Services for each task so that the overall QoS and cost requirements of the composition are satisfied. A travel agency application is considered to facilitate dynamic service composition, selection and adaptation in order to compose and to adjust the travel process to meet tourists' needs regardless of their locations, platforms and/or hardware speeds. The proposed Gravitational Search Algorithm based on user preference in the Web Service Selection is obtaining better results. The future work may be achieve towards incorporations other evolutionary Computing Algorithm the same.

\section{REFERENCES}

[1]. Al-Masri E., Mahmoud Q. H.,(2007) "Discovering the best web service", (poster) 16th Intl. Conference on World Wide Web (WWW), 2007, pp. 1257-1258. (For QWS Dataset Version 1.0 or QWS Dataset Version 2.0).

[2]. Canfora G.,( Penta M., Di,Esposito R., and Villani M.,(2004) "A lightweight approach for QoS-aware service composition", In Proc. of the 2nd Intl. Conference on Service Oriented Computing, New York, USA. 2004.

[3]. Demian Antony D’Mello, Ananthanaranyana. “A Review of Quality of Service (QoS) Driven Dynamic Web Service Selection Techniques" ICIIS 2010, Jul 29Aug 01,2010, India.

[4]. Demian Antony D' Mello, V.S.Ananthanaranyana., (2009) "A Tree Structure for Web Service Composition”.@2009.

[5]. Dmytro Zhovtobryukh., "A Petri Net-based Approach for Automated Goal-Driven Web Service Composition". Simulation 2007; 83; 33.

[6]. Esmat Rashedi, Hossien Nezamabadi-pour, Saeid Saryazdi, Malihe M. Farsangi. (2010) "Allocation of Static Var Compensator Using Gravitational Search Algorithm".IEEE Conference on Web Serivce 2010.

[7]. Junsun, BinFeng, Wenboxu, "Particle swarm Optimization with particles having Quantum Behavior".

[8]. Joyce El Haddad, Maude Manouvrier, and Marta Rukoz, (2010) "TQoS: Transactional and QoS-Aware Selection Algorithm for Automatic Web Service Composition", IEEE Transactions on Services Computing, Vol. 3,No. 1.

[9]. Kennedy,I. and Eberhart.,R. "Particle swarm Optimization", Pmc.IEEE int.Conf. On Neural Network, 1995: 1942-1948.

[10]. Prashanth,B. and Narahari,Y. "Efficient Algorithm for Combinatorial Auctions with Volume Discounts Arising in Web Service Composition".

[11]. Manoharan,R. Archana,A. and Siddhika Cowlagi(2011) "Hybrid Web Services Ranking Algorithm". IJCSI, Vol 8, Issue 3, No.2, May 2011. 
[12]. Ming Chen, Zhen-wu Wang, (2007) “An Approach for Web Services Composition Based on QoS and Discrete Particle Swarm Optimization", ACIS Intl. Conference on Software Engineering, AI, Networking, and Parallel/Distributed Computing, 2007, vol. 2, pp. 37-4.

[13]. Martine De Cock, Sam Chung, Omar Haffez, (2007) "Selection of Web Services with Imprecise QoS Constraints", Proceedings of the IEEE/WIC/ACM International Conference on Web Intelligence.

[14]. Rashedia,E. Nezamabadi-pour,H. and Saryazdi,S. (2009) "GSA: A Gravitational Search Algorithm", Elsevier, New York, NY, USA, June 2009, pp. 22322248.

[15]. Su Myeon Kim, Marcel-Catalin Rosu., (2004) "A Survey of Public Web Services". www 2004, May 17-22, 2004, New York, USA.

[16]. Shuzhi LI, Ping SHEN, Shux in YANG, "A Grouping Particle Swarm Optimization Algorithm for web service based on user Preference".

[17]. W3C Working Group. Web services architecture. http://www.w3.org/.

[18]. Wei-Hua Ai, Yun-Xian Huang, Hui Zhang, and Ning Zhou, (2008) "Web Services Composition and Optimizing Algorithm Based on QoS", 4th Intl. Conference on Wireless Communications, Networking and Mobile Computing, 2008. WiCOM '08, Oct. 2008.

[19]. Zibanezhad,B. Zamanifar,K. Nematbakhsh,N. Mardukhi,F. (2009) “An Approach for Web Services Composition Based on QoS and Gravitational Search Algorithm”.Whitepaper Microsoft copyrights@2009.

[20]. Zhang Liang-Jie, Li Bing, and Chao Tian st al., (2003) "On demand Web services-based business process composition", In Proc. of the IEEE Intl. Conference on System, Man, and Cybernetics, Washington, USA, 2003. 